Published in: Computational Science and Its Applications, (A. H. Siddiqi, R. C. Singh, G. D. Veerappa Gowda, eds.), Taylor and Francis Group, 2020, pp. 41-59.

Personal file

\title{
A Model to Assess the Role of Spatial Urban Configurations on Crowd Evacuation Dynamics During Terrorist Attacks
}

D. Provitolo, (Université Côte d'Azur, CNRS, Observatoire de la Côte d'Azur, IRD, Géoazur, France, damienne.provitolo@geoazur.unice.fr )

R. Lozi, (Université Côte d'Azur, CNRS, LJAD, France, Rene.LOZI@univ-cotedazur.fr)

E. Tric, (Université Côte d'Azur, CNRS, Observatoire de la Côte d'Azur, IRD, Géoazur,

France, emmanuel.tric@univ-cotedazur.fr)

\section{CONTENTS}

3.1 Introduction: Modern Terrorist Attacks

3.2 Weighted PCR System: A Model for Analyzing the Dynamics of Human

Behavior During a Disaster

3.2.1 Network of Squares and Streets Modelling Spatial Urban Configurations

3.2.2 Equations of the WPCR System on Each Node 6

3.2.3 Transitional Dynamics $\quad 7$

3.3 Coupled WPCR System on a Network 9

3.3.1 Flows and Bottleneck Coupling 9

3.3.2 Fixed Points of the Three-Node System 13

3.4 Influence of the Spatial Configuration on the Pace of Evacuation 18

3.4.1 Scaling the Parameters 18

3.4.2 Influence of the Intermediate Place Capacity on the Evacuation Dynamics $\quad 18$

3.5Conclusion 20

Acknowledgments 20

$\begin{array}{ll}\text { References } & 20\end{array}$

\subsection{Introduction: Modern Terrorist Attacks}

Terrorism has always existed in history and has taken different forms: anarchist attacks, political attacks, or attacks by colonial states, such as the terrible Jallianwala Bagh massacre, which took place on April 13, 1919 in Amritsar, Punjab, when a crowd of non-violent protesters were fired upon by troops of the British Indian Army.

In this article we focus specifically on behavior of populations facing new forms of terrorism, such as the attacks perpetrated by sects, groups or "lone wolves" that are becoming more and more prevalent. Three types of acts can be distinguished: 
- shooting and bombing attacks, for example in bus and subway stations (Madrid, Spain, 2004; Paris, France, 1995, 2015; Nigeria, 2014; Belgium, 2016; Kashmir, 2019), at airports (Brussels airport, 2016; Atatürk airport, 2016), in urban areas with simultaneous terrorist acts (Mumbai, India, 2008, with 12 coordinated shooting and bombing attacks lasting four days).

- attacks using CBRN (chemical, biological, radiological, and nuclear weapons) such as the sarin gas attack on the Tokyo subway committed by the Aum sect (March 20, 1995) (Dauphiné and Provitolo 2013).

- in response to tightened police controls aiming to reduce bombing attacks, new types of ad hoc weapons are now being employed, such as suicide planes on the World Trade Center (2001), lorries or cars that are simply driven directly through a crowd massed for a cultural event or strolling peacefully. These attacks are most often led by "lone wolves", who are difficult to detect beforehand and equally difficult to locate because they are "nested" among civilians. On July 14, 2016, 86 people who attended the Bastille Day fireworks on the "Promenade des Anglais" in Nice, France, were killed; 458 more were wounded.

These attacks, perpetrated in crowds, in frequented places, or on public transport at rush hour, give rise to diversified human reactions. One can observe behaviors of sideration (paralysing panic), of flight panic, of agitation in all directions, of reasoned flight, of sheltering, or mutually offered assistance (Crocq 2013), etc.

Except for sideration, sheltering and escape behaviors, whether taken under the influence of panic (flight panic) or of reason (reasoned escape), produce trajectories of displacement. When an attack happens, the topography of the area is also very important. The dynamics of human reactions and the associated displacements are indeed guided by the space and the alternatives that it offers, as displayed in Figure 3.1, especially in terms of evacuation, flight, and accessibility to refuge areas, such as safe places and squares.

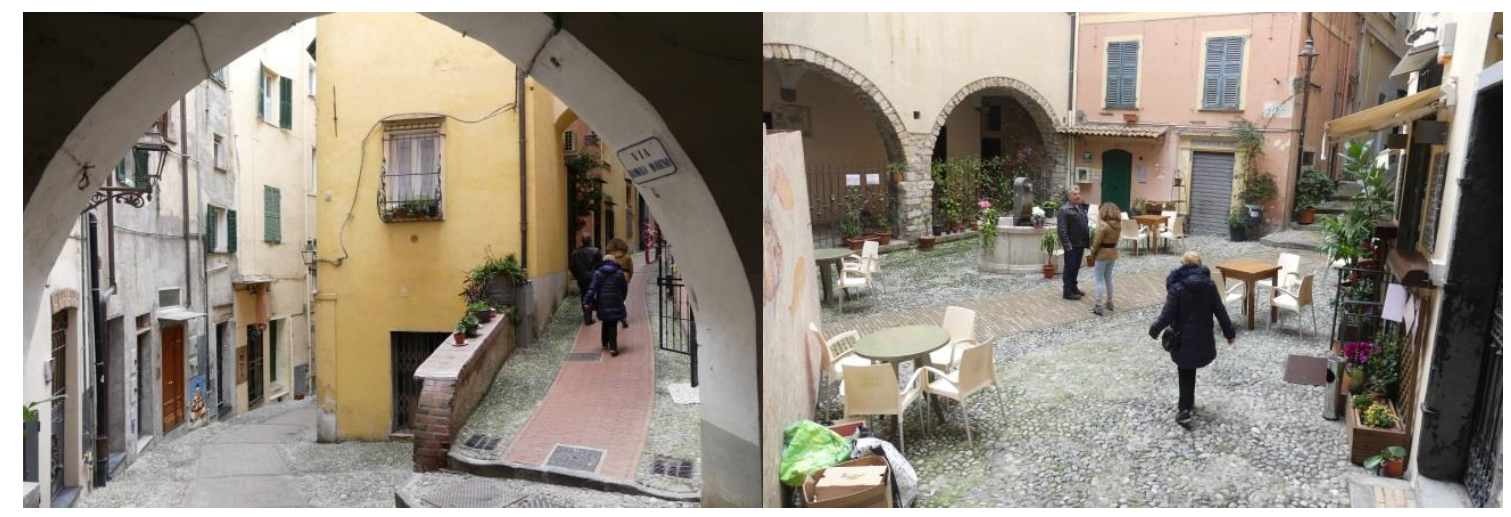

FIGURE 3.1

Street and places in Mediterranean city of Italy (@ D. Provitolo, March, 2019.)

There are few data available to identify the dynamics of the displacements associated with 
these behaviors. Of course, it is difficult to artificially reproduce a disaster, which would otherwise allow us to observe the diversity of human reactions that could occur, to follow the spatio-temporal dynamics, and to analyze the impact of spatial configurations on these dynamics. To overcome these limits, it is possible to develop mathematical models from which hypotheses are tested by means of a human behavior computer simulation model.

The Com $2 \mathrm{SiCa}$ research team ${ }^{1}$ proposed the Panic-Control-Reflex (PCR) model (Provitolo et al. 2015) as shown in Figure 3.2, which is a model that simulates the possible human behavior that can occur during sudden onset and unpredictable disasters, such as terrorist attacks. This model is formed by a system of ordinary differential equations to describe behavioral dynamics over time (Verdière et al. 2014, 2015, Cantin et al. 2016).

Neuroscience research explains that during a disaster, humans are rarely stuck in one type of behavior. The population switches between different behavioral states, some of which are the result of instinctive, others of reasoned, reaction.

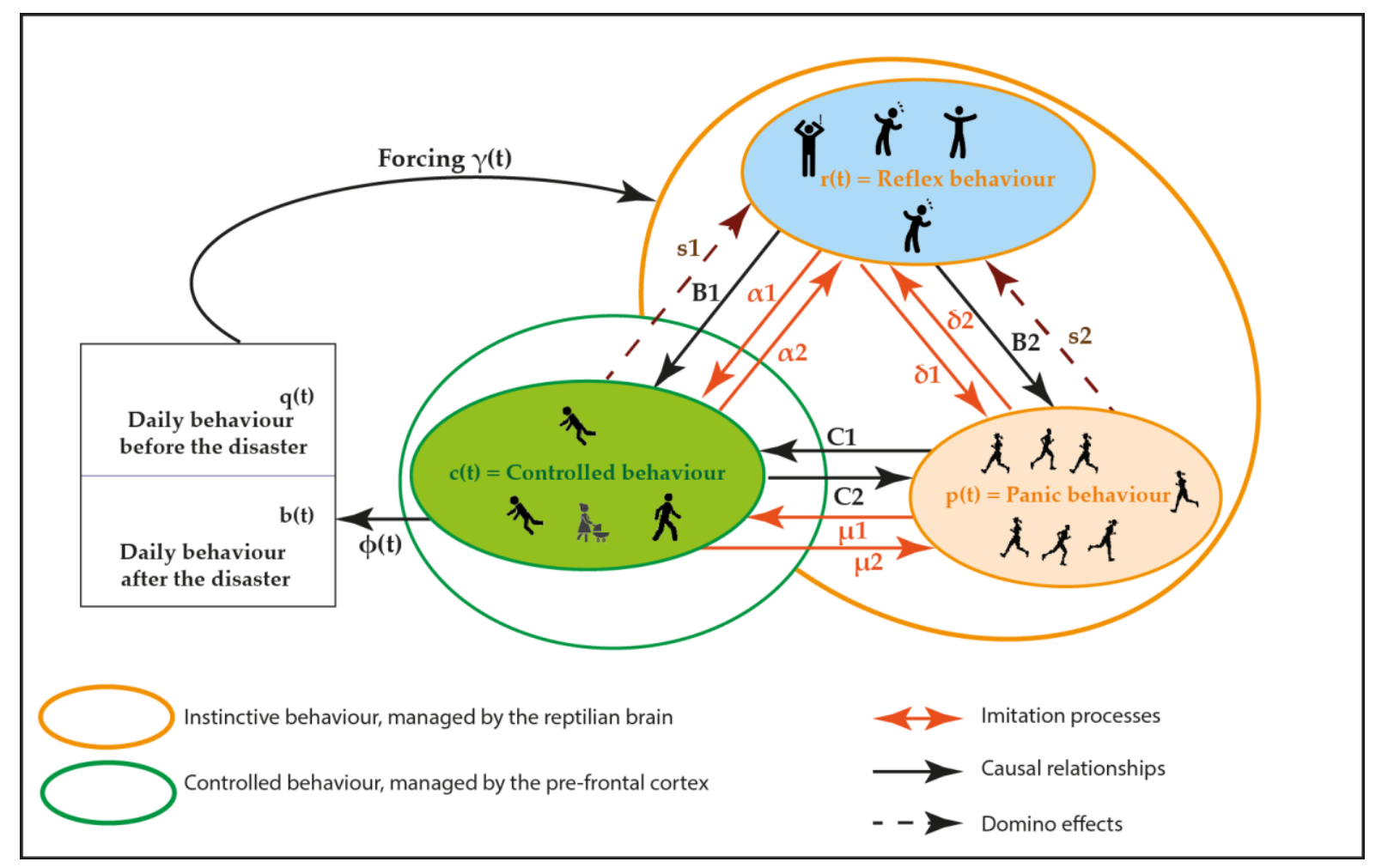

FIGURE 3.2

Graphic representation of the Panic-Control-Reflex behavior model (PCR) in the exceptional situation of disaster. (Source: Provitolo et al. 2015).

As the brain switches from one behavioral state to another, in the context of terrorist

\footnotetext{
${ }^{1}$ https://geoazur.oca.eu/en/research-geoazur/2158-com2sica-how-to-comprehend-and-simulate-humanbehaviors-in-areas-facing-natural-disasters
} 
attacks and therefore in a situation of sudden and unforeseen threat, the entire impacted population first adopts a behavioral reaction, Reflex $r(t)$, under the influence of surprise and the suddenness of the event, before transiting to the Panic reflex behavior $p(t)$, or Controlled behavior $c(t)$ (Provitolo et al. 2015). Reflex $r(t)$ and Panic $p(t)$ behavior are managed by the reptilian brain: they are instinctive, automatic reactions, allowing one to react extremely quickly to the threat, either by being stunned and paralyzed $r(t)$, or by fleeing as quickly as possible due to the panic fear $p(t)$. Controlled behavior $c(t)$ is managed by the pre-frontal cortex, which concerns reasoned and self-control reactions. These can take different forms during a catastrophe, for example, in the form of evacuation, escape, containment, sheltering, search for help, mutual aid, or, on the contrary, looting, etc. Despite their diversity, the PCR model aggregates all of these controlled behaviors.

In this article we propose an extension of the PCR model in order to take into account the influence of spatial configuration in the mathematical modeling of the dynamics of human reactions in the face of traumatic situations, such as terrorist attacks. We call this extended model the Coupled Weighted PCR (CWPCR).

In Section 3.2, we will present the Weighted Panic Control Reflex model in its graphic and mathematical form, as published by the Com2SiCa research team. This model is improved from the PCR model in order to take into account the role of spatial configurations on behavioral dynamics. In Section 3.3 we introduce the CWPCR model, which incorporates the pressure and counterpressure of the crowd in each place via a bottleneck effect, which takes into account the narrowness and the length of the streets and the size of places. We will consider an oriented network with three nodes representing three places or public squares of different sizes, linked by narrow streets. In this network, we compute the equilibrium points that show the motion of the crowd after a terrorist attack. Section 3.4 is therefore devoted to the analysis of the impact of the size of the intermediate place of this network on the evacuation of the population in the face of a terrorist attack, by means of numerical simulations. The numerical results highlight that, depending on their respective size, intermediate places modulate the dynamics and the speed of flow of the crowd. In this sense, they become strategic places, both for the planners who must think about the organization of the area to host public events and festivals, and also for the terrorists who can use these strategic places to multiply the effect of their harmful actions by trapping the escape movements between two areas of action. Finally, in Section 3.5, a brief conclusion will be drawn.

\subsection{Weighted PCR System: A Model for Analyzing the Dynamics of Human Behavior During a Disaster}

\subsubsection{Network of Squares and Streets Modelling Spatial Urban Configurations}

Just before a dramatic event like a terror attack in a city, the crowd is generally spread across several places, public squares and streets. In the aftermath of the initial shock, people rush through the streets to reach what they think will be more secure places, as one can see in 
Figure 3.3.

In this chapter, we define a city by a mathematical graph, where the places and public squares are called vertices or nodes and are denoted $N_{1}, N_{2}, \cdots, N_{p}$, and the streets, escalators, doors, and stairs are the oriented edges $\left(N_{i} \rightarrow N_{j}\right)$ linking these nodes. They are oriented, because the flow from one place toward another is not symmetric during terrorist attacks. Our aim is to model the motion of the crowd through such edges. To achieve this, we must introduce some "geographical" particularities of the city, like the size of places, the narrowness of streets, and the number of people initially present in each place. This is why we need to upgrade the standard PCR model, into the Weighted PCR (WPCR) model, by introducing new data, with Weight standing for the relative sizes of crowd, places, and streets.

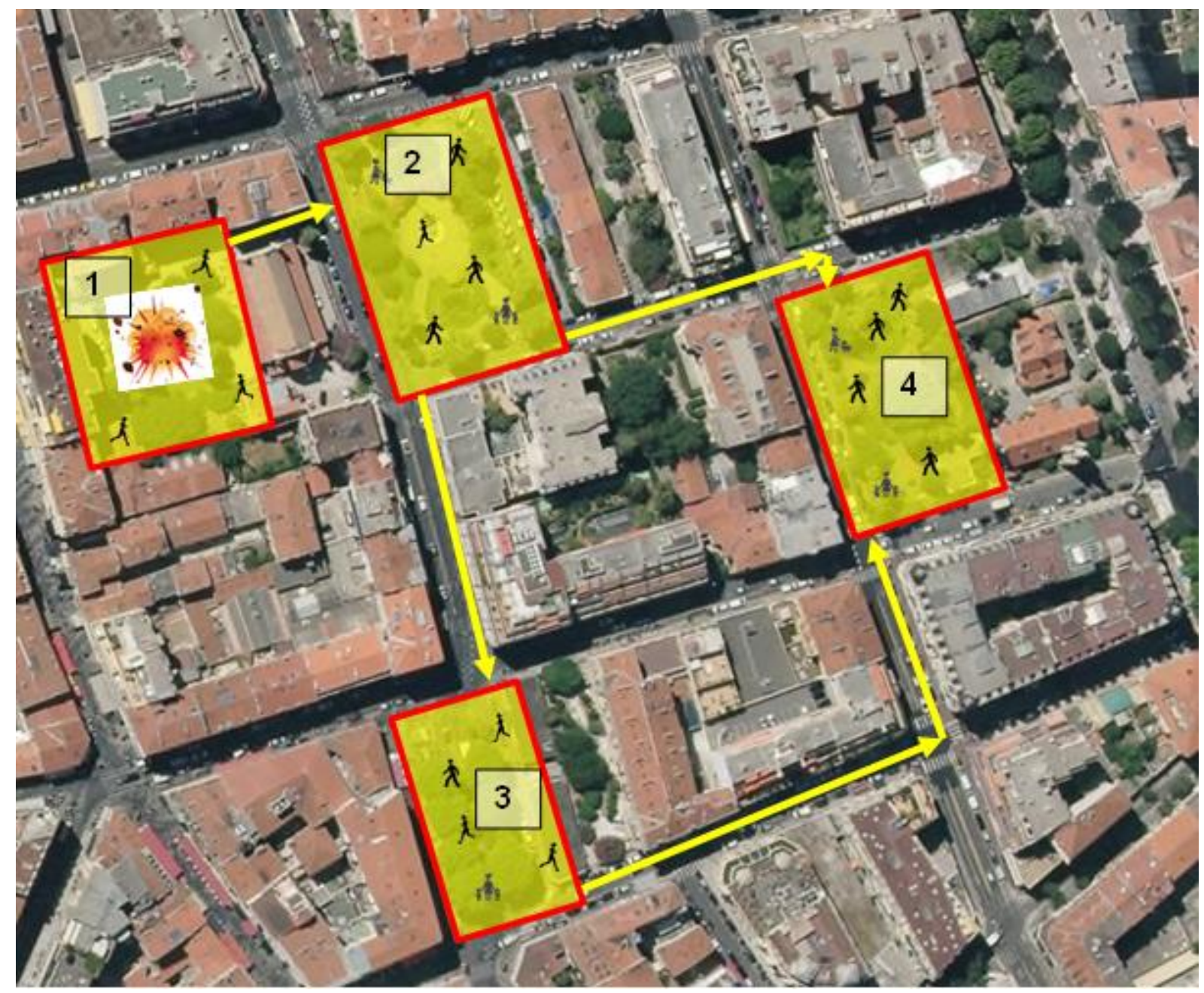

\section{FIGURE 3.3}

Possible paths of rushing people in a city network. (IGN - BD Orthophoto, 2017, $50 \mathrm{~cm}$ resolution).

First, on each node $N_{k}, \quad k=1, p$ we call $r_{k}(t), c_{k}(t), p_{k}(t), q_{k}(t), b_{k}(t)$ the number of people being in reflex, control, panic, daily usual behavior, and return normal life and $V_{k}(t)$ the total number of people present at this node 


$$
V_{k}(t)=r_{k}(t)+c_{k}(t)+p_{k}(t)+q_{k}(t)+b_{k}(t)
$$

Second, in order to more precisely model the characteristics of the city, we introduce $W_{k}$, the maximum capacity of the number of people who can be present in each node $N_{k}$ (i.e. due to the size of the corresponding place). Each maximum capacity is a constant. Each time one must have

$$
V_{k}(t) \leq W_{k}
$$

\subsubsection{Equations of the WPCR System on Each Node}

The WPCR simulates the human behavior, in one specific place, during a catastrophic event. As explained in the introduction, it is based on neuroscience studies. During the event, the switches from one behavioral state to another are caused by transitional dynamics, as schematized in Figure 3.2 due to:

i. Causal relationships $\left(B_{1}, B_{2}, C_{1}, C_{2}\right)$. Once the population is in the reflex behavior state, a part of it can evolve toward controlled behaviors at the rate $B_{1}$, while another part transitions towards panic behaviors at the rate $B_{2}$. Likewise, a part of the panicked population may switch to controlled behavior at the rate $C_{l}$. According to the evolution of the situation, individuals who have adopted a controlled behavior may switch back to panic behavior at the rate $C_{2}$. This process can be iterated many times.

ii. Processes of imitation and contagion, which are well known in crowd psychology and have been termed "emotional contagion" (Hatfield et al. 1994). The imitation processes are modelled identically to epidemiological propagation (Provitolo 2005). The imitation is valid in both directions and is modelled by the function $F(r, c)$ (see Equation (3.5)) for emotional contagion between reflex and controlled behavior (using the damping coefficients $\alpha_{1}$ and $\alpha_{2}$ ), by the function $G(r, p)$ (see Equation (3.6)) for emotional contagion between reflex and panic behavior $\left(\delta_{1}\right.$ and $\left.\delta_{2}\right)$, and by the function $H(c, p)$ (see Equation (3.7)) for emotional contagion between controlled and panicked behavior $\left(\mu_{1}\right.$ and $\left.\mu_{2}\right)$.

iii. Domino effects, which illustrate a succession of events $\left(s_{1}\right.$ and $\left.s_{2}\right)$ correspond, for example, to a new attack in an urban area or to a closed door during an evacuation. In the PCR model, the parameters $s_{1}$ and $s_{2}$ are either constant or built in a periodic form.

The (WPCR) model is defined on each node $k$ by

$$
\dot{X}=\Phi(t, X)
$$

with $\dot{X}=\frac{d X}{d t}, X=(r, c, p, q, b)^{T} \in \mathbb{R}^{5}$ and $\Phi$ given by $\Phi(t, X)=\left(\Phi_{i}(t, X)\right)^{T}, i=1, \ldots, 5$, where the functions $\Phi_{i}$ are defined by 


$$
\left\{\begin{aligned}
\Phi_{1}\left(t, X_{k}\right)=\dot{r}_{k}(t)= & \gamma(t) q_{k}(t)\left(W_{k}-r_{k}(t)\right)-\left(B_{1}+B_{2}\right) r_{k}(t)+s_{1}(t) c_{k}(t)+s_{2}(t) p_{k}(t) \\
& +F\left(r_{k}(t), c_{k}(t)\right) r_{k}(t) c_{k}(t)+G\left(r_{k}(t), p_{k}(t)\right) r_{k}(t) p_{k}(t) \\
\Phi_{2}\left(t, X_{k}\right)=\dot{c}_{k}(t)= & -\varphi(t) c_{k}(t)\left(W_{k}-b_{k}(t)\right)+B_{1} r_{k}(t)+C_{1} p_{k}(t)-C_{2} c_{k}(t)-s_{1}(t) c_{k}(t) \\
& -F\left(r_{k}(t), c_{k}(t)\right) r_{k}(t) c_{k}(t)+H\left(c_{k}(t), p_{k}(t)\right) c_{k}(t) p_{k}(t) \\
\Phi_{3}\left(t, X_{k}\right)=\dot{p}_{k}(t)= & B_{2} r_{k}(t)-C_{1} p_{k}(t)+C_{2} c_{k}(t)-s_{2}(t) p_{k}(t)-G\left(r_{k}(t), p_{k}(t)\right) r_{k}(t) p_{k}(t) \\
& -H\left(c_{k}(t), p_{k}(t)\right) c_{k}(t) p_{k}(t) \\
\Phi_{4}\left(t, X_{k}\right)= & \dot{q}_{k}(t)=-\gamma(t) q_{k}(t)\left(W_{k}-r_{k}(t)\right) \\
\Phi_{5}\left(t, X_{k}\right)= & \dot{b}_{k}(t)=\varphi(t) c_{k}(t)\left(W_{k}-b_{k}(t)\right)
\end{aligned}\right.
$$

The parameters involved in Equation (3.4) are real positive coefficients:

$$
B_{i} \geq 0, C_{i} \geq 0, i=1,2 ; \alpha_{i} \geq 0, \delta_{i} \geq 0, \mu_{i} \geq 0, i=1,2 ; s_{i} \geq 0, i=1,2 .
$$

The imitation functions $F, G$ and $H$ are real valued functions defined on $\mathbb{R} \times \mathbb{R}$ by

$$
\begin{aligned}
& F(r(t), c(t))=-\alpha_{1} f_{1}\left(\frac{r(t)}{c(t)+\varepsilon}\right)+\alpha_{2} f_{2}\left(\frac{c(t)}{r(t)+\varepsilon}\right) \\
& G(r(t), p(t))=-\delta_{1} g_{1}\left(\frac{r(t)}{p(t)+\varepsilon}\right)+\delta_{2} g_{2}\left(\frac{p(t)}{r(t)+\varepsilon}\right) \\
& H(c(t), p(t))=\mu_{1} h_{1}\left(\frac{c(t)}{p(t)+\varepsilon}\right)-\mu_{2} h_{2}\left(\frac{p(t)}{c(t)+\varepsilon}\right),
\end{aligned}
$$

where $\varepsilon$ is a positive number and $f_{i}, g_{i}, h_{i}$ for $i=1,2$ are real valued functions defined on $\mathbb{R}$. They have a decreasing shape indicating that the behavior imitation is symmetric. Moreover they are normalized,

$$
0 \leq f_{i}(u) \leq 1,0 \leq g_{i}(u) \leq 1,0 \leq h_{i}(u) \leq 1, \quad \forall u \in R, i=1,2
$$

Because this model does not take the mortality rate into account, the population remains constant. Therefore Equation (3.4) is considered when time is proceeding from an initial time $t_{0} \geq 0$, with initial condition

$$
\begin{gathered}
r_{k}\left(t_{0}\right)+c_{k}\left(t_{0}\right)+p_{k}\left(t_{0}\right)+q_{k}\left(t_{0}\right)+b_{k}\left(t_{0}\right)=V_{k}\left(t_{0}\right)= \\
=r_{k, 0}+c_{k, 0}+p_{k, 0}+q_{k, 0}+b_{k, 0}=V_{k, 0} \leq W_{k}
\end{gathered}
$$

that satisfies the following properties

$$
r\left(t_{0}\right)>0, c\left(t_{0}\right)>0, p\left(t_{0}\right)>0, q\left(t_{0}\right)>0, b\left(t_{0}\right)>0
$$

We suppose that the characteristic parameters $B_{1}, B_{2}, C_{1}, C_{2}$, of each node have the same value, because they depend on cultural and psychological factors specific to each individual rather than to spatial configurations and crowd context. It is why we use function $\Phi_{i}$ instead of function $\Phi_{k, i}$.

\subsubsection{Transitional Dynamics}

Both forcing functions, $\gamma$ and $\varphi$ respectively, model the beginning of the disaster and the return to a quiescent daily behavior. Their shape can be adapted to various scenarios. When $t$ is sufficiently large, they satisfy $\gamma(t)=\varphi(t)=1$. In catastrophic situations it is considered 
that $\gamma$ is a stiff function, ranging from 0 to 1 in a very brief interval of time (Verdière et al. 2014, 2015, Cantin et al. 2016) because if we consider a bomb attack, the entire crowd that is near the explosion passes from daily to reflex behavior in an instant, and it takes a very long time for people to return to their normal state.

Therefore, one can suppose that a terror attack is shaped by two characteristic times: $t_{s}$ (for start) and $t_{e}$ (for end) with $t_{0}<t_{s}<t_{e}$ for which

$$
\left\{\begin{array}{l}
\gamma(t)=1, \forall t \geq t_{s} \\
\varphi(t)=0, \forall t<t_{e}
\end{array}\right.
$$

As an example, for $I_{\text {trans }}=[3.2,43.2]$, these functions can be defined by

$$
\begin{aligned}
& \varphi(t)=\left\{\begin{aligned}
0 \text { if } & 0 \leq x<43.2 \\
\cos ^{2}\left(2 \pi \frac{x-3.2}{160}\right), & \text { if } 43.2 \leq x \leq 83.2, \\
1, & \text { if } x>83.2
\end{aligned}\right. \\
& \gamma(t)=\left\{\begin{aligned}
\cos ^{2}\left(2 \pi \frac{x-3.2}{12.8}\right), & \text { if } 0 \leq x \leq 3.2, \\
1, \quad \text { if } & x>3.2
\end{aligned}\right.
\end{aligned}
$$

There are displayed in Figure 3.4.

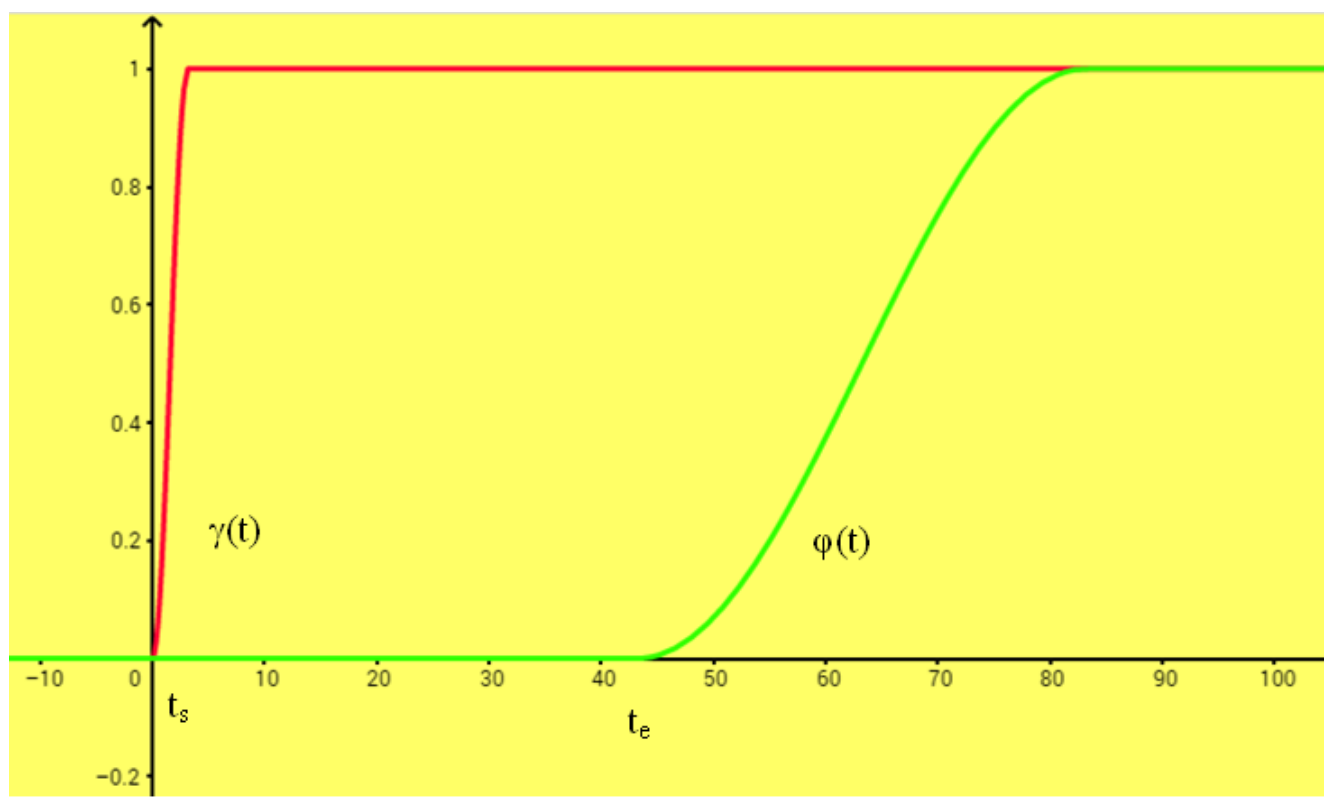

\section{FIGURE 3.4}

Forcing functions $\gamma(t)$, and $\varphi(t), I_{\text {trans }}=[3.2,43.2]$

Following Cantin et al. (2016) we keep the term transitional dynamics for the dynamics of the PCR model (likewise for both improved WPCR, and CWPCR model presented in Section 3.3 ) in the interval of time $I_{\text {trans }}=\left[t_{s}, t_{e}\right]$ (in terror attacks this interval of time can last from several minutes up to hours, as observed during the 2016 terrorist attack in Nice). Therefore in $\forall t \in I_{\text {trans }}$ functions $\varphi$ and $\gamma$ verify 


$$
\left\{\begin{array}{l}
\gamma(t)=1 \\
\varphi(t)=0
\end{array}\right.
$$

Hence, during the transitional dynamics, the population with daily behavior collapses and there is not yet a population that is back to daily behavior (i.e. $q(t)=b(t)=0$ ).

System (3.4) is reduced to

$$
\left\{\begin{aligned}
\dot{r}(t)= & -\left(B_{1}+B_{2}\right) r(t)+s_{1}(t) c(t)+s_{2}(t) p(t)+ \\
& F(r(t), c(t)) r(t) c(t)+G(r(t), p(t)) r(t) p(t) \\
\dot{c}(t)= & B_{1} r(t)+C_{1} p(t)-C_{2} c(t)-s_{1}(t) c(t)- \\
& F(r(t), c(t)) r(t) c(t)+H(c(t), p(t)) c(t) p(t) \\
\dot{p}(t)= & B_{2} r(t)-C_{1} p(t)+C_{2} c(t)-s_{2}(t) p(t)- \\
& G(r(t), p(t)) r(t) p(t)-H(c(t), p(t)) c(t) p(t) .
\end{aligned}\right.
$$

\subsection{Coupled WPCR system on a network}

\subsubsection{Flows and bottleneck coupling}

As previously defined, nodes are linked by edges. We now aim to model the motion of the crowd through such edges (i.e. streets, stairs, escalators, doors, etc.).

We also seek to identify the obstacles that slow the escape of the crowd in the aftermath of the initial shock by analyzing the topology of the network of streets and places in a city. This information can potentially be used to improve the design of a city to facilitate the escape of a crowd towards more secure places.

For the sake of simplicity, as we show in Figure 3.5, we consider first a simplified oriented network with only three nodes $\left(N_{1} ; W_{1}\right) ;\left(N_{2} ; W_{2}\right) ;\left(N_{3} ; W_{3}\right)$ and two edges $\left(N_{1} \rightarrow\right.$ $\left.N_{2}\right) ;\left(N_{2} \rightarrow N_{3}\right)$. Such a simplified network can be straightforwardly complexified by adding as many nodes and edges as necessary, without any difficulty. However, it is better to first focus our attention on the nature of the obstacles in this simplified network.

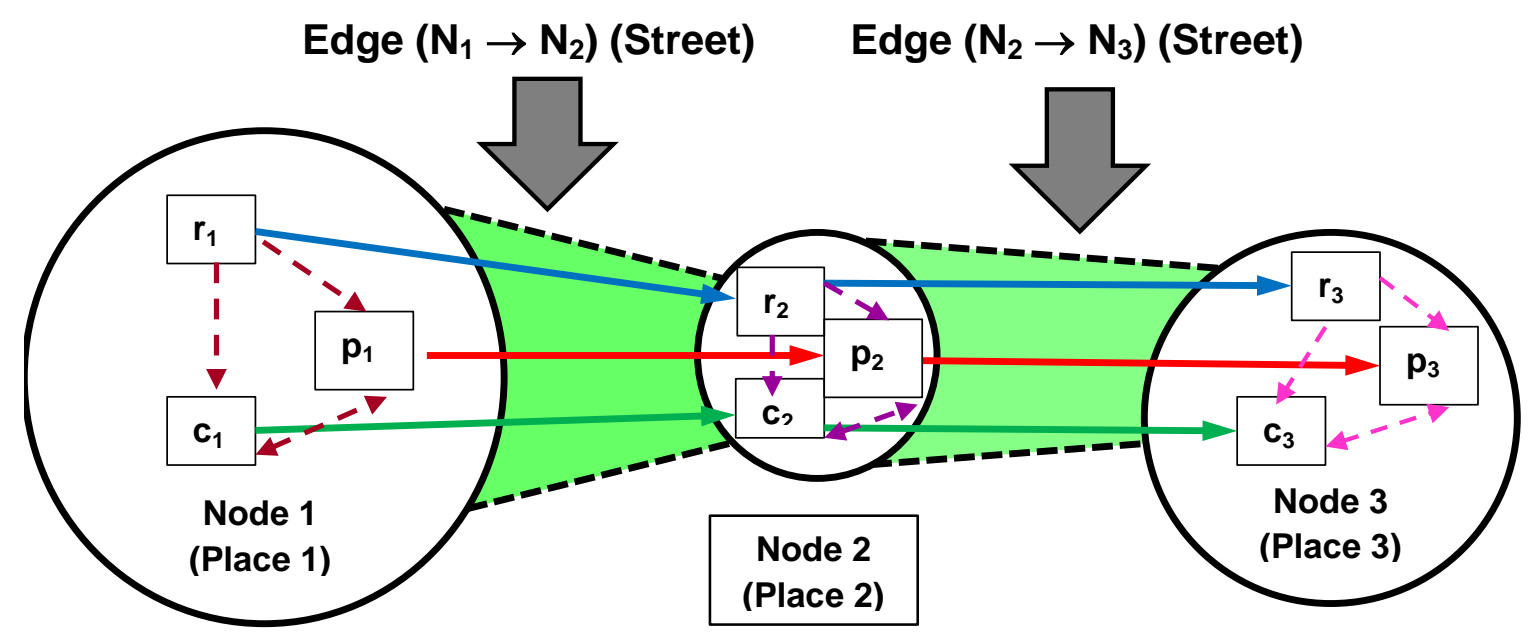

FIGURE 3.5

Three node network: people in every state are rushing from node 1 towards node 2, and from node 2 towards node 3 , keeping the same behavioral class. 
We suppose that during the short interval of time when people are traveling inside one edge, they remain in the same behavioral class as displayed in Figure 3.5. In this figure, the "geographical" edges $\left(N_{1} \rightarrow N_{2}\right) ;\left(N_{2} \rightarrow N_{3}\right)$ (i.e. the streets linking node 1 to node 2 , and node 2 to node 3) are split in three "behavioral edges" meaning that on the same street people in reflex, panic, or controlled behavior are escaping from node 1 to node 2 (and from node 2 to node 3 ), therefore people in each particular behavior in node 1 are meeting people in the same behavior in node 2 (and in the same manner from node 2 to node 3 ).

To continue focusing on the special coupling that we are introducing here, we suppose that imitation mechanisms are not activated (i.e. $F \equiv H \equiv G \equiv 0$, which is equivalent to $\alpha_{i}=$ $\delta_{i}=\mu_{i}=0$ for $\left.i=1,2\right)$, and, furthermore, there is no domino effect $\left(s_{i}=0, i=1,2\right)$ and we also suppose that we are in the interval $I_{\text {trans }}=\left[t_{s}, t_{e}\right]$ where only transitional dynamics are considered. Of course, it is easy to relax such limitations, which are not dependent upon the coupling, by not eliminating the corresponding terms in the equations.

In each node $N_{k}, k=1,2,3$ such transitional dynamics are the solution of the system

$$
\left\{\begin{array}{l}
\dot{r}_{k}(t)=-\left(B_{1}+B_{2}\right) r_{k}(t) \\
\dot{c}_{k}(t)=B_{1} r_{k}(t)+C_{1} p_{k}(t)-C_{2} c_{k}(t) \\
\dot{p}_{k}(t)=B_{2} r_{k}(t)-C_{1} p_{k}(t)+C_{2} c_{k}(t)
\end{array}\right.
$$

which is the reduction of System (3.4) in the transition interval (and a particular case of Equation (3.15)).

Note: in the WPCR model, the terms $\dot{r}_{k}(t), \dot{c}_{k}(t), \dot{p}_{k}(t), \dot{q}_{k}(t), \dot{b}_{k}(t)$ can be considered as flows, because a flow is a quantity of something divided by a unit of time. There are two kinds of flow. In Equations (3.4), (3.15), (3.16) flows are "behavioral", as they represent the quantities of people changing their behavior per unit of time. Now we consider also "motion" flows, which are the quantities of people in each behavior class, moving from one node to another node, per unit of time. Of course, both kinds of flow are combined to produce a global equivalent in the following equations.

Many studies on pedestrian flows have been published (Daamen et al. 2005, Dias et al. 2013, Kretz et al. 2006, Liao et al. 2014, Seyfried et al. 2005, Zhang et al. 2013, Zhou et al. 2014). We consider, in particular, the survey of Daamen et al. (2005), in which the graphs of six different experiments showing the relationship between the flow of pedestrians and their densities are displayed. All these graphs show clearly a nonlinear relationship of a logistic type between density and flow.

Moreover, Daamen and co-authors developed a first-order traffic flow theory to describe two-dimensional pedestrian flow operations in the case of an oversaturated bottleneck in front of which a large, high-density region has formed. Such a mathematical model also highlights the logistic relationship for any bottleneck width as one can see in Figure 3.6. 


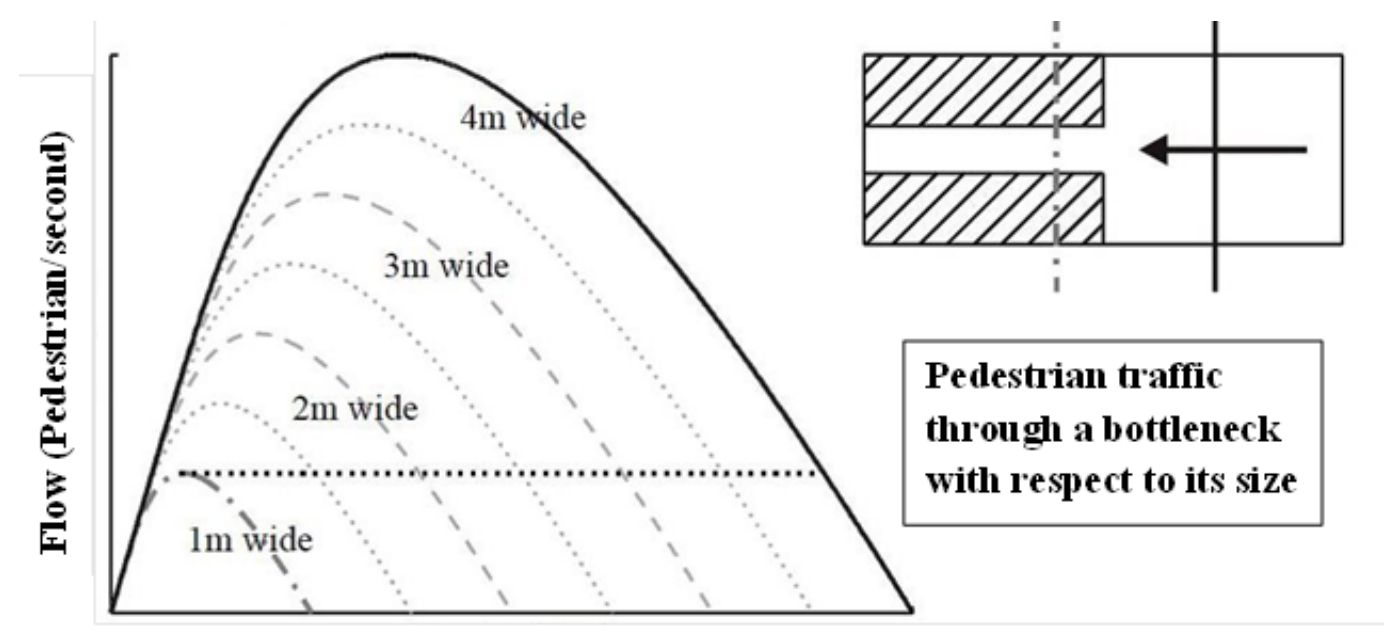

Density (Pedestrian/ meter)

\section{FIGURE 3.6}

Relationship between flow and density of pedestrians going through an oversaturated bottleneck. (Adapted from Daamen et al. 2005).

We now introduce our hypothesis for the coupled WPCR (CWPCR), based on this type of logistic relationship. Again, for the sake of simplicity, we consider only unidirectional motion of the crowd (i.e. motion on an oriented graph), as the one displayed in Figure 3.5, because it is supposed that a terrorist attack occurs in node 1 and that people try to escape from this node towards nodes 2 and 3.

When the crowd is moving from one node to another, its speed and the corresponding motion flow depends on three factors. The first factor reflects the narrowness and the length of the street. More people can go from one place to the next if the street is large, rather than in the case of a narrow street. This topological characteristic will be modelled by "roughness" coefficients $\eta_{1,2}, \eta_{2,3}$. In fact, in the considered coupling, we suppose that people cannot change their behavior when they move from one node to another (e.g. controlled people remain controlled, panicked people remain panicked, and so on), and thus we use three such roughness coefficients $\eta_{c, 1,2}, \eta_{p, 1,2}, \eta_{r, 1,2}, \eta_{c, 2,3}, \eta_{p, 2,3}, \eta_{r, 2,3}$ to refer to the population in reflex, panic, or controlled situations, respectively. Of course, they can have the same value. The second factor is proportional to the number of people present in the node from which the crowd is escaping. This is equivalent to the pressure of the crowd in these places. The third factor reflects the counterpressure due to the maximal capacity of the place toward which the people are fleeing, conjugated with the number of persons already present there. The combination of pressure and counterpressure gives a bottleneck effect.

We propose to model this bottleneck effect by a nonlinearity of a logistic type to keep the same philosophy as Daamen et al. (2005).

Thus, the bottleneck coupling corresponding to the one of Figure 3.5, is given by the system 


$$
\begin{aligned}
& \left(\begin{array}{l}
\dot{r}_{1}(t)=-\left(B_{1}+B_{2}\right) r_{1}(t)-\eta_{r, 1,2} r_{1}(t)\left(W_{2}-c_{2}(t)-p_{2}(t)-r_{2}(t)\right) \\
\dot{c}_{1}(t)=B_{1} r_{1}(t)+C_{1} p_{1}(t)-C_{2} c_{1}(t)-\eta_{c, 1,2} c_{1}(t)\left(W_{2}-c_{2}(t)-p_{2}(t)-r_{2}(t)\right) \\
\dot{p}_{1}(t)=B_{2} r_{1}(t)-C_{1} p_{1}(t)+C_{2} c_{1}(t)-\eta_{p, 1,2} p_{1}(t)\left(W_{2}-c_{2}(t)-p_{2}(t)-r_{2}(t)\right)
\end{array}\right. \\
& \dot{r}_{2}(t)=-\left(B_{1}+B_{2}\right) r_{2}(t)+\eta_{r, 1,2} r_{1}(t)\left(W_{2}-c_{2}(t)-p_{2}(t)-r_{2}(t)\right) \\
& -\eta_{r, 2,3} r_{2}(t)\left(W_{3}-c_{3}(t)-p_{3}(t)-r_{3}(t)\right) \\
& \dot{c}_{2}(t)=B_{1} r_{2}(t)+C_{1} p_{2}(t)-C_{2} c_{2}(t)+\eta_{c, 1,2} c_{1}(t)\left(W_{2}-c_{2}(t)-p_{2}(t)-r_{2}(t)\right) \\
& -\eta_{c, 2,3} c_{2}(t)\left(W_{3}-c_{3}(t)-p_{3}(t)-r_{3}(t)\right) \\
& \dot{p}_{2}(t)=B_{2} r_{2}(t)-C_{1} p_{2}(t)+C_{2} c_{2}(t)+\eta_{p, 1,2} p_{1}(t)\left(W_{2}(t)-c_{2}(t)-p_{2}(t)-r_{2}(t)\right) \\
& -\eta_{p, 2,3} p_{2}\left(W_{3}-c_{3}(t)-p_{3}(t)-r_{3}(t)\right) \\
& \dot{r}_{3}(t)=-\left(B_{1}+B_{2}\right) r_{3}(t)+\eta_{r, 2,3} r_{2}(t)\left(W_{3}-c_{3}(t)-p_{3}(t)-r_{3}(t)\right) \\
& \dot{c}_{3}(t)=B_{1} r_{3}(t)+C_{1} p_{3}(t)-C_{2} c_{3}(t)+\eta_{c, 2,3} c_{2}(t)\left(W_{3}-c_{3}(t)-p_{3}(t)-r_{3}(t)\right) \\
& \dot{p}_{3}(t)=B_{2} r_{3}(t)-C_{1} p_{3}(t)+C_{2} c_{3}(t)+\eta_{p, 2,3} p_{2}(t)\left(W_{3}-c_{3}(t)-p_{3}(t)-r_{3}(t)\right)
\end{aligned}
$$

with initial conditions satisfying

$$
\begin{aligned}
& r_{1,0}+c_{1,0}+p_{1,0}=V_{1,0} \leq W_{1} ; r_{2,0}+c_{2,0}+p_{2,0}=V_{2,0} \leq W_{2} ; r_{3,0}+c_{3,0}+p_{3,0}= \\
& V_{3,0} \leq W_{3}
\end{aligned}
$$

In this system the bottleneck coupling, concerning, for example, the controlled population that is moving from node 1 to node 2 is given by the term:

$$
\eta_{c, 1,2} c_{1}(t)\left(W_{2}-c_{2}(t)-p_{2}(t)-r_{2}(t)\right)
$$

in the second equation of (3.17)

$$
\dot{c}_{1}(t)=B_{1} r_{1}(t)+C_{1} p_{1}(t)-C_{2} c_{1}(t)-\eta_{c, 1,2} c_{1}(t)\left(W_{2}-c_{2}(t)-p_{2}(t)-r_{2}(t)\right)
$$

In this bottleneck coupling (3.19), $\eta_{c, 1,2}$ is the parameter that models the topological characteristic of the street linking node 1 to node 2. The second factor $c_{1}(t)$ of Equation (3.19) reflects the pressure of controlled people in node 1 willing to escape towards node 2 and also the proportionality of people escaping with respect to people staying in node 1 . Finally, the factor $\left(W_{2}-c_{2}(t)-p_{2}(t)-r_{2}(t)\right)$ shows the counterpressure which is maximum (i.e. the term vanishes) when $W_{2}=c_{2}(t)+p_{2}(t)+r_{2}(t)$ because, in this case, there is no more room for people coming from node 1 . This bottleneck coupling is nonlinear, as shown on Figure 3.7. 


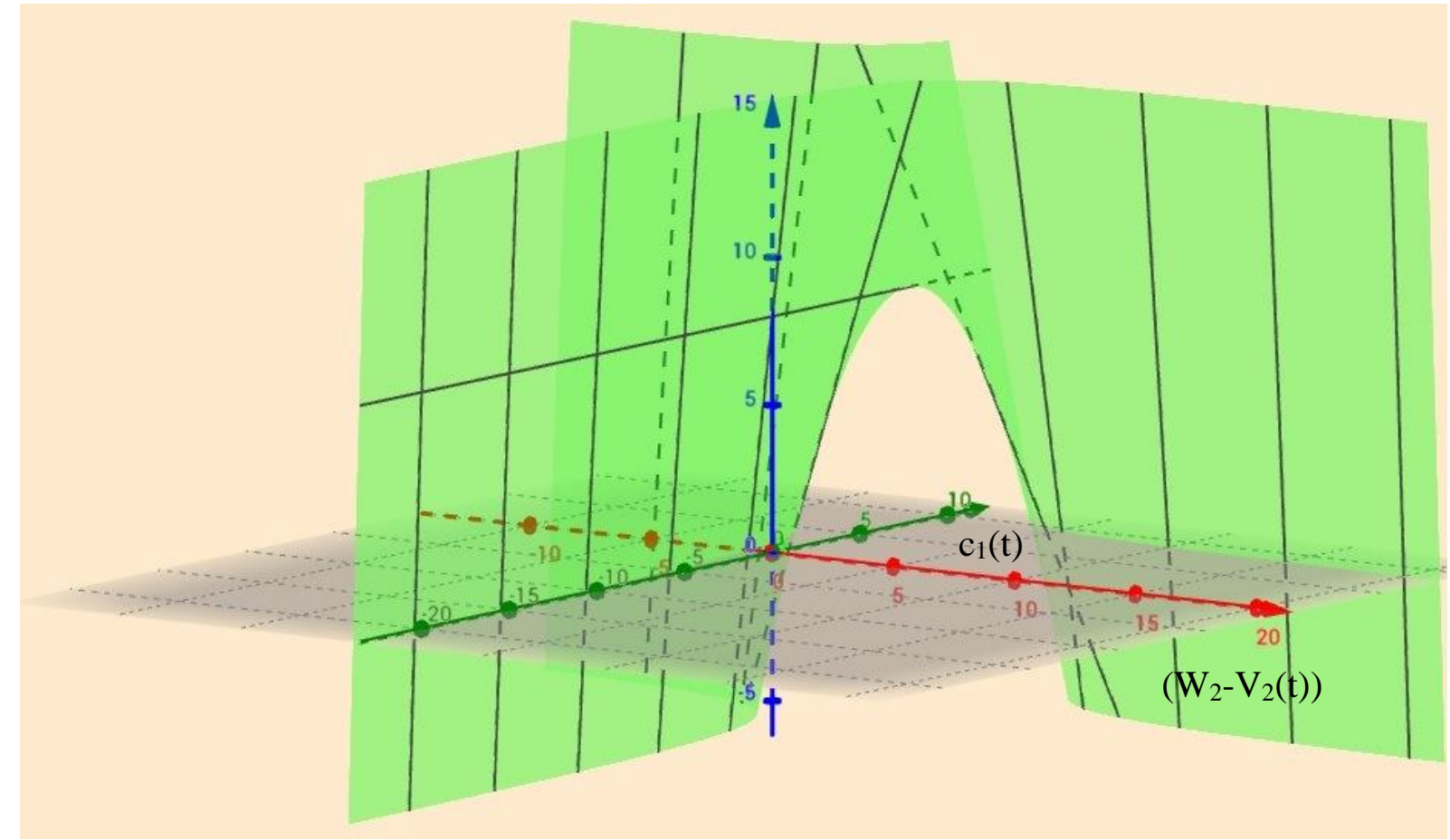

FIGURE 3.7

Graph of the bottleneck coupling function.

As we consider only transitional dynamics where $q_{k}(t)=b_{k}(t)=0$, Equation (3.19) can be written

$$
\eta_{c, 1,2} c_{1}(t)\left(W_{2}-V_{2}(t)\right)
$$

\subsubsection{Fixed points of the three-node system}

The fixed-point research allows us to identify the point of equilibrium towards which the system tends during the transitional period. This equilibrium point highlights the primordial role of the size of both nodes 2 and 3 in the context of evacuation dynamics. It is important to note that the mathematically calculated equilibrium point does not necessarily correspond to the equilibrium situation sought by crisis management personnel. The population stranded in the initial place (node 1) remains very vulnerable to the terrorist threat.

To stay closer to reality, we further assume that people in reflex situation are stunned and paralyzed. They cannot rush from one node to another as indicated in Figure 3.8. Therefore, the transitions between $r_{1}, r_{2}, r_{3}$ are forbidden. People in this stunned state can only change their behavior (from $r_{k}$ to $p_{k}$ or $c_{k}$ ). 


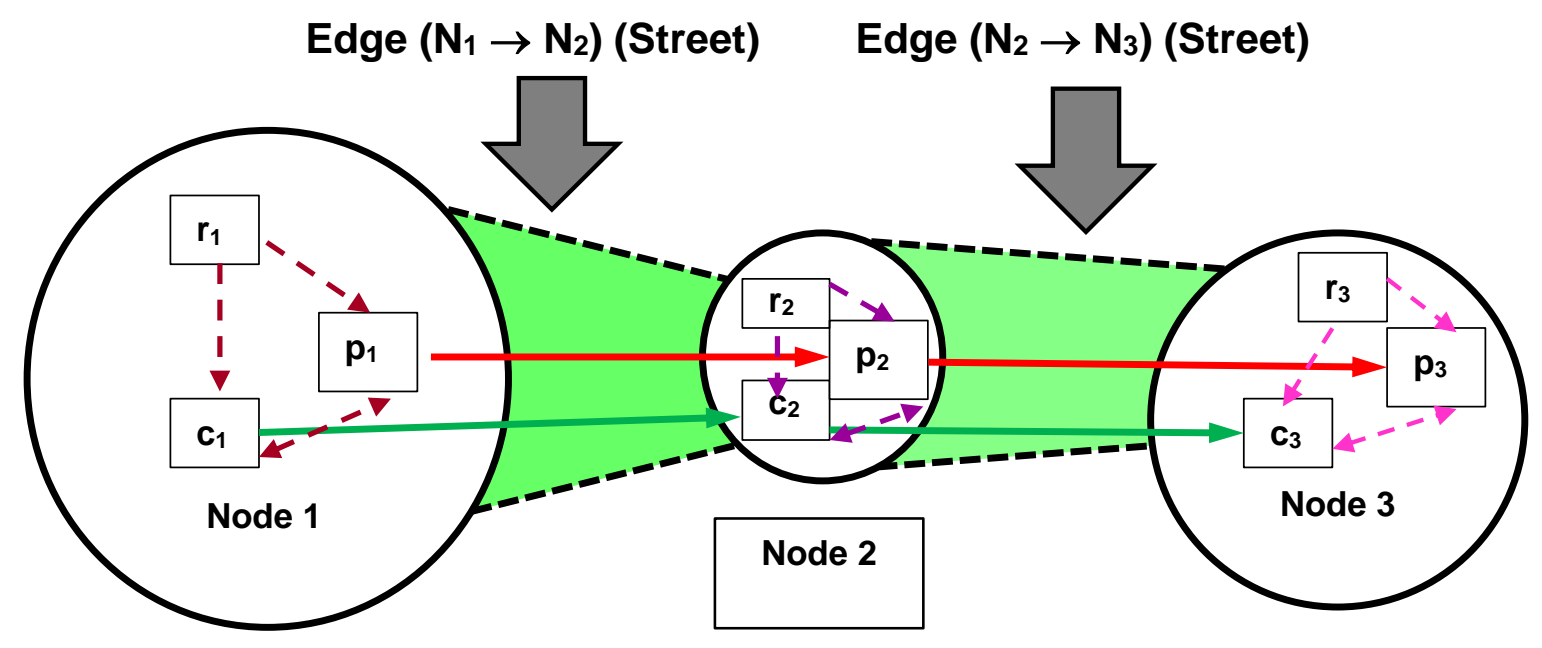

\section{FIGURE 3.8}

Three node network: people in the reflex behavior state are stranded in their original node.

To achieve this goal Equation (3.17) is simply modified to vanish parameters $\eta_{r, i, j}, i=1,2, j=i+1$.

The fixed point $\left(r_{1}^{*}, c_{1}^{*}, p_{1}^{*}, r_{2}^{*}, c_{2}^{*}, p_{2}^{*}, r_{3}^{*}, c_{3}^{*}, p_{3}^{*}\right)$ of the system toward which the solution of Equation (3.17) converges, is straightforwardly computed.

$$
\left\{\begin{array}{l}
r_{1}^{*}=0 \\
c_{1}^{*}=0 \\
p_{1}^{*}=0 \\
r_{2}^{*}=0 \\
c_{2}^{*}=0 \\
p_{2}^{*}=0 \quad \text { if } \quad V_{1,0}+V_{2,0}+V_{3,0} \leq W_{3}, \\
r_{3}^{*}=0 \\
c_{3}^{*}=\frac{C_{1}\left(V_{1,0}+V_{2,0}+V_{3,0}\right)}{C_{1}+C_{2}} \\
p_{3}^{*}=\frac{C_{2}\left(V_{1,0}+V_{2,0}+V_{3,0}\right)}{C_{1}+C_{2}}
\end{array}\right.
$$




$$
\begin{aligned}
& \left\{\begin{array}{l}
r_{1}^{*}=0 \\
c_{1}^{*}=0 \\
p_{1}^{*}=0 \\
r_{2}^{*}=0 \\
c_{2}^{*}=\frac{C_{1}\left(V_{1,0}+V_{2,0}+V_{3,0}-W_{3}\right)}{C_{1}+C_{2}} \\
p_{2}^{*}=\frac{C_{2}\left(V_{1,0}+V_{2,0}+V_{3,0}-W_{3}\right)}{C_{1}+C_{2}} \\
r_{3}^{*}=0 \\
c_{3}^{*}=\frac{C_{1} W_{3}}{C_{1}+C_{2}} \\
p_{3}^{*}=\frac{C_{2} W_{3}}{C_{1}+C_{2}}
\end{array}\right. \\
& \left\{\begin{array}{l}
r_{1}^{*}=0 \\
c_{1}^{*}=\frac{C_{1}\left(V_{1,0}+V_{2,0}+V_{3,0}-W_{2}-W_{3}\right)}{C_{1}+C_{2}} \\
p_{1}^{*}=\frac{C_{2}\left(V_{1,0}+V_{2,0}+V_{3,0}-W_{2}-W_{3}\right)}{C_{1}+C_{2}} \\
r_{2}^{*}=0 \\
c_{2}^{*}=\frac{C_{1} W_{2}}{C_{1}+C_{2}} \\
p_{2}^{*}=\frac{C_{2} W_{2}}{C_{1}+C_{2}} \\
r_{3}^{*}=0 \\
c_{3}^{*}=\frac{C_{1} W_{3}}{C_{1}+C_{2}} \\
p_{3}^{*}=\frac{C_{2} W_{3}}{C_{1}+C_{2}}
\end{array}\right.
\end{aligned}
$$

The values of this fixed point mean that:

Situation 1: if the number of people initially staying in the three nodes is less than the capacity of node 3 (i.e. $V_{1,0}+V_{2,0}+V_{3,0} \leq W_{3}$ ), after a while, both nodes 1 and 2 become empty and all the crowd has sought refuge in node 3 .

Alternatively, if this number is greater than the capacity (i.e. $V_{1,0}+V_{2,0}+V_{3,0}>W_{3}$ ), then two situations can occur:

Situation 2: node 3 becomes full and the remaining people are still stranded in node 2 if $V_{1,0}+V_{2,0}+V_{3,0} \leq W_{2}+W_{3}$

or, Situation 3: stranded in both nodes 1 and 2 if $V_{1,0}+V_{2,0}+V_{3,0}>W_{2}+W_{3}$.

From Equations (3.21), (3.22), (3.23) it is obvious that only the ratio $\frac{C_{1}}{C_{2}}$ is significant for the limit of solutions of Equation (3.17) because $\frac{c_{1}^{*}}{p_{1}^{*}}=\frac{c_{2}^{*}}{p_{2}^{*}}=\frac{c_{3}^{*}}{p_{3}^{*}}=\frac{C_{1}}{C_{2}}$ (when defined), instead of parameters $B_{1}$ and $B_{2}$, becomes important for the pace at which the "reservoir" of people in reflex behavior is emptied. 
Of course, across the world there are different cultures, which lead to different behaviors. These behaviors can be modelled varying parameters.

For example, if populations are not made aware of major risks and not prepared for them, it causes a panic reaction $\left(B_{1}<B_{2}\right)$, this behavior is then regulated by the ratio $\frac{C_{1}}{C_{2}}$. The higher this ratio, the more the population remains or transits in the controlled state.

In simulated situation, we choose a set of parameters that highlight a weak risk culture, while favoring the return to a controlled behavior: the values, $B_{1}=0.15, B_{2}=0.45$, mean that there is a weak risk culture and, $C_{1}=0.21, C_{2}=0.07$, mean that the panic in the crowd context is compensated by controlled reactions for a part of the population who keep selfcontrol, notably because there is no new threat or sudden attack.

Therefore, in situation 1 when $V_{1,0}=18000, V_{2,0}=0, V_{3,0}=0, W_{2}=1000, W_{3}=$ 18500 and $\eta_{c, 1,2}=\eta_{c, 2,3}=\eta_{p, 1,2}=\eta_{p, 2,3}=0.004$, one obtains the following convergence towards the fixed point:

$$
\begin{gathered}
r_{1}^{*}=0, c_{1}^{*}=0, p_{1}^{*}=0, r_{2}^{*}=0, c_{2}^{*}=0, p_{2}^{*}=0, \\
r_{3}^{*}=0, c_{3}^{*}=\frac{C_{1}\left(V_{1,0}+V_{2,0}+V_{3,0}\right)}{C_{1}+C_{2}}=\frac{0.21(18000)}{0.21+0.07}=13500, p_{3}^{*}=\frac{C_{2}\left(V_{1,0}+V_{2,0}+V_{3,0}\right)}{C_{1}+C_{2}}=4500 .
\end{gathered}
$$

This value means that, initially, all the people are in node 1 and both nodes 2 and 3 are empty, but after a certain period of time, both nodes 1 and 2 are empty and everyone has reached node 3 . One can see the flow of people through the two edges $\left(N_{1} \rightarrow N_{2}\right) ;\left(N_{2} \rightarrow\right.$ $N_{3}$ ), in Figure 3.9. 


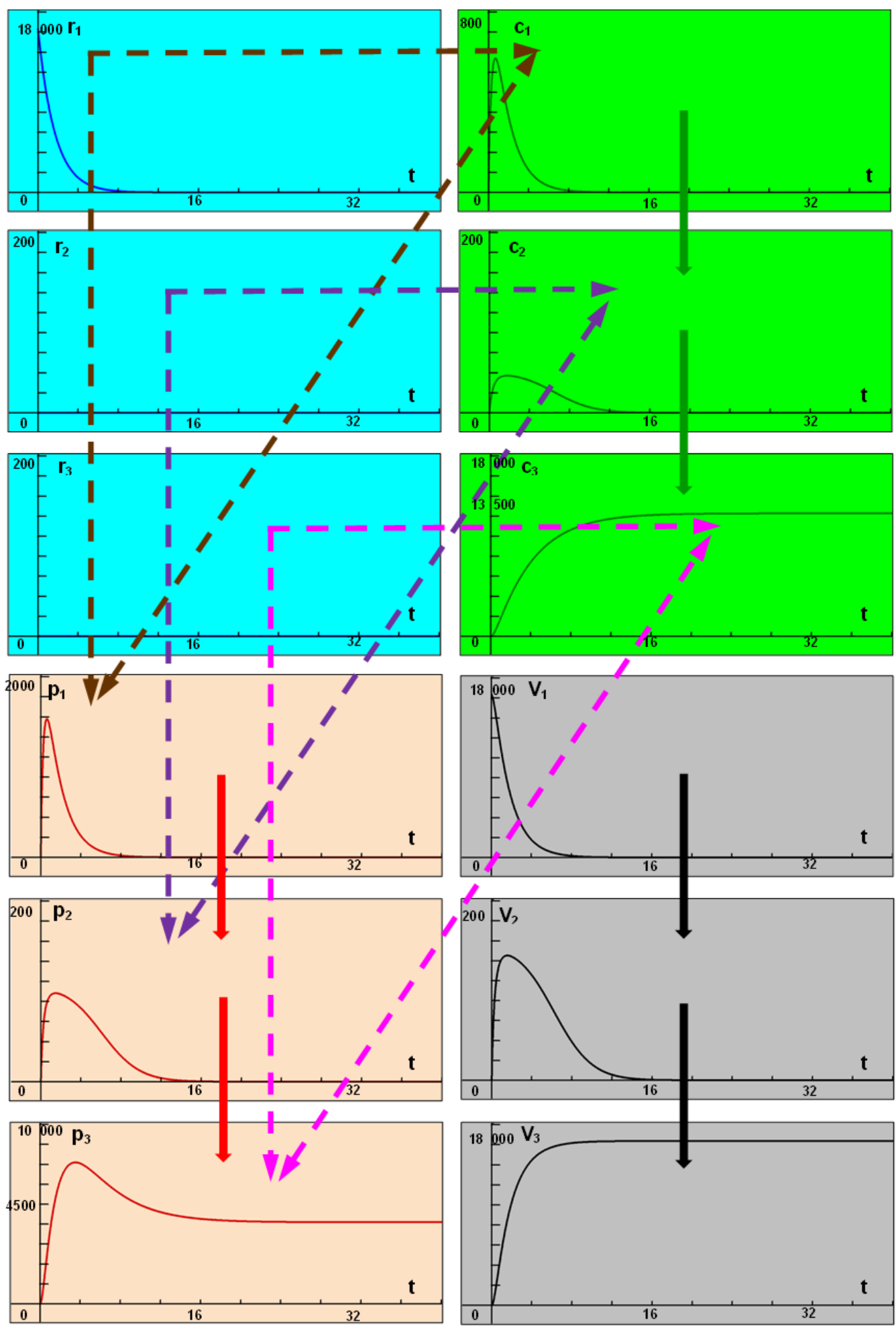

\section{FIGURE 3.9}

Situation 1: Convergence towards the fixed point $(0,0,0,0,0,0,0,13500,4500)$. In this figure the change of behavioral states is symbolized by dotted arrows, and the motion between nodes by plain arrows is as displayed in Figure 3.8. 


\subsection{Influence of the spatial configuration on the pace of evacuation}

We now seek to identify the obstacles that slow the escape of the crowd in the aftermath of the initial shock by analyzing the topology of the network of streets and places in a city. This information can potentially be used to improve the design of a city to facilitate the escape of a crowd towards more secure places.

\subsubsection{Scaling the parameters}

Fixing the value of all the parameters in PCR, WPCR or CWPCR is a very complicated task, which has not yet been done on an experimental basis. In the framework of the Com $2 \mathrm{SiCa}$ project, an experimental protocol is under scrutiny, in order to achieve these results in the near future. However, it is important to note that only relative values between parameters are important, because there is a relationship between the unit of time and the unit used for the parameters. In other words, considering all the parameters of the adimensional Equation (3.17), (i.e. $B_{i} \geq 0, C_{i} \geq 0, \eta_{c, i, j}, \eta_{p, i, j}, \eta_{r, i, j}, i=1,2, j=i+1$ ) integrated with respect to the variable time $t$, it is nearly equivalent to consider such parameters multiplied by the same constant $\kappa$ and integrated using the time variable $\tau=\frac{t}{\kappa}$ (e.g. $t$ can be considered in seconds, minutes, or hours). There is not, strictly speaking, equivalence because such parameters are linearly used in the WPCR model, however in the CWPCR model the coupling is nonlinear and a slight distortion intervenes during a transient short period for some variables.

\subsubsection{Influence of the intermediate place capacity on the evacuation dynamics}

Intermediate places play a central role in the fluidity or, to the contrary, the congestion of movement between a dangerous place and a place of shelter. This can be shown in the following numerical experiments: with the same parameter values, except for the size of node 2 , we analyse the speed at which the people are emptying the place of the terrorist (node 1).

As previously described (Section 3.3.2), we consider the values of the parameters $B_{1}=0.15, B_{2}=0.45, C_{1}=0.21, C_{2}=0.07$ and we choose $\eta_{c, 1,2}=\eta_{c, 2,3}=\eta_{p, 1,2}=$ $\eta_{p, 2,3}=0.004$.

We consider the following values of $W_{2}: 50,100,200,1000$ for which we display the dynamics in Figure 3.10. 

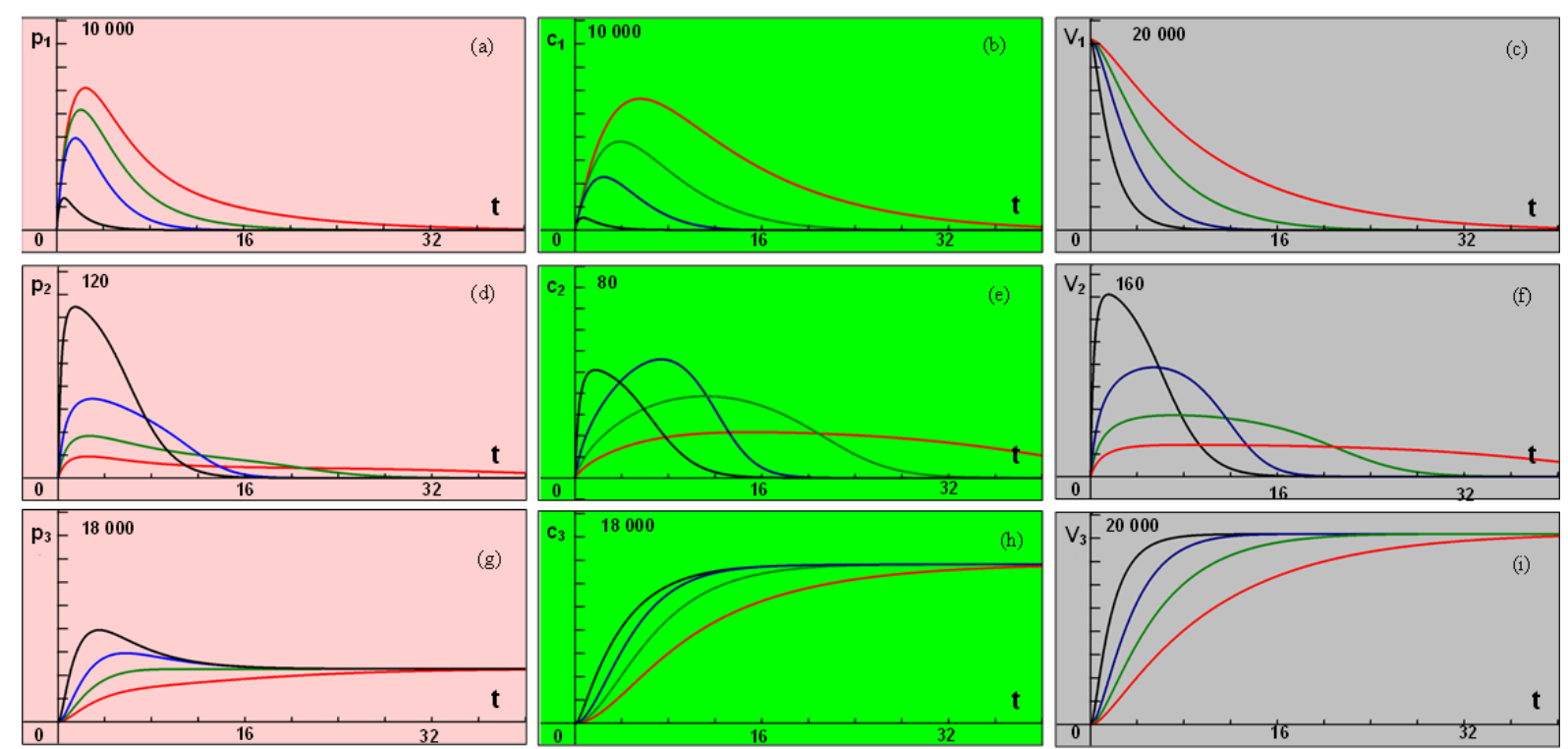

FIGURE 3.10

Situation 1: Convergence towards the fixed point $(0,0,0,0,0,0,0,13500,4500)$ for the values of parameters $B_{1}=0.15, B_{2}=0.45, C_{1}=0.21, C_{2}=0.07, \eta_{c, 1,2}=\eta_{c, 2,3}=\eta_{p, 1,2}=\eta_{p, 2,3}=0.004, \quad V_{1,0}=$ $18000, V_{2,0}=0, V_{3,0}=0, W_{3}=18500$, and the values of $W_{2}=50$ (red curves), $W_{2}=100$ (green curves), $W_{2}=200$ (blue curves), $W_{2}=1000$ (black curves).

In the case $W_{2}=1000$ (black curves) the flight of the entire population from place 1 to place 2 (which can be a small square) and then to place 3 is very fast; it lasts less than 10 minutes (Figures 3.10c, f, i), because place 3 can accommodate the entire population. There is a massive influx of panicked people (Figure 3.10d), which is greater than the controlled one (Figure 3.10e), into place 2, which empties very quickly as the majority of controlled people reach the safe shelter (Figure 3.10h). However, it can be noted that a significant number of the panicked population remains in the refuge place, and this number is only slowly decreased (see bump in Figure 3.10g).

This is explained by the fact that the flight dynamics are not hindered by obstacles or bottlenecks, and the fleeing populations have not enough time to change their behavioural state.

On the other hand, if $W_{2}=50$ (red curves), the evacuation of the total population from place 1 to place 2 and then place 3 is much slower (about 35 minutes instead of less than 10 minutes, (Figures $3.10 \mathrm{c}, \mathrm{f}, \mathrm{i})$ ). The panicked population has time to calm down in place 1, because there is no new attack (it has been assumed that there is no domino effect, $\left(s_{i}=\right.$ $0, i=1,2)$ ). It is thus a population that is mainly in a state of reasoned behavior that arrives in place 3 (Figures $3.10 \mathrm{~g}, \mathrm{~h}$ ).

It can be highlighted from this first analysis that the faster the speed of change of location (hence decreasing the vulnerability of populations), the faster this speed leads to significant flows of panic in both places 2 and 3. There is a paradox here: the fast self-safety movement of populations leads to situations of collective panic that are more difficult to manage. This fact must be taken into account by emergency services and emergency physicians. 
Although the deaths that occur are not included in this CWPCR model version, one can imagine that the escape of panicked populations would give rise to more victims.

As has already been said, the simulation results shed light on the importance of the size of the intermediate places and their role in the fluidity or, to the contrary, on the congestion of movements between a dangerous place and a place of shelter.

Depending on their respective size, intermediate places will modulate the dynamics and the speed of flow of the crowds. In this sense, they become strategic places both for the planners, who must think about the organization of the area to host public events, and also for the terrorists who can use these strategic places to multiply the effect of their harmful actions by trapping the escape movements between two areas of action.

\subsection{Conclusion}

In this article we have developed a new model of weighted human behavior coupled with street and place networks, in the context of an urban terrorist attack, thus improving the PCR model with bottleneck coupling and by taking into account the capacity of each place and the number of people stranded in these places. The simulation results in a simple network with three nodes (places or public squares) and two edges (streets) that demonstrates the key role of the capacity of an intermediate place in the dynamics of evacuation from dangerous to safe places.

\section{Acknowledgements}

This work has been supported by the French government, through i) the National Research Agency (ANR) under the Societal Challenge 9 "Freedom and security of Europe, its citizens and residents" with the reference number ANR-17-CE39-0008, co-financed by French Defence Procurement Agency (DGA) and The General Secretariat for Defence and National Security (SGDSN), and ii) the UCA ${ }^{\mathrm{JEDI}}$ Investments in the Future project managed by the National Research Agency (ANR) with the reference number ANR-15-IDEX-01.

\section{References}

Cantin, G., Verdière, N., Lanza, V., et al. 2016. Mathematical Modeling of Human Behaviors During Catastrophic Events: Stability and Bifurcations. International Journal of Bifurcation and Chaos Vol. 26 No. 10:1630025 (20 p.).

Crocq, L. 2013. Les paniques collectives. Paris: Odile Jacob.

Daamen, W., Hoogendoorn, S. P., Bovy, P. H. L. 2005. First-Order Pedestrian Traffic Flow. Theory. Transp. Res. Rec. J. Transp. Res. Board 1934:43-52.

Dauphiné, A., Provitolo, D. 2013. Risques et catastrophes-Observer, spatialiser, comprendre, gérer. Paris : A. Colin ( $2^{\text {ème }}$ édition), Collection U.

Dias, C., Sarvi, M., Shiwakoti, N., Ejtemai, O., Burd, M. 2013. Investigating collective escape behaviors in complex situations. Safety Science 60:87-94. 
Hatfield, E., Cacioppo, J. T. and Rapson, R. L. 1994. Emotional Contagion. Cambridge: Cambridge University Press.

Kretz, T., Grünebohm, A. and Schreckenberg, M. 2006. Experimental study of pedestrian flow through a bottleneck. Journal of Statistical Mechanics: Theory and Experiment Volume 2006:P10014.

Liao, W., Seyfried, A., Zhang, J., Boltes, M., Zheng, X., Zhao, Y. 2014. Experimental study on pedestrian flow through wide bottleneck. The Conference on Pedestrian and Evacuation Dynamics 2014 (PED2014). Transportation Research Procedia 2:26 - 33.

Provitolo, D. 2005. Un exemple d'effets de dominos : la panique dans les catastrophes urbaines. Cybergéo : Revue européenne de géographie $\mathrm{n}^{\circ}$ 328:19 p. http://www.cybergeo.eu/index2998.html

Provitolo, D., Dubos-Paillard, E., Verdière, et al. 2015. Les comportements humains en situation de catastrophe: de l'observation à la modélisation conceptuelle et mathématique. Cybergeo : Européean Journal of Geography [on line], document 735:23 p.

Seyfried, A., Steffen, B., Klingsch, W., and Boltes, M. 2005. The Fundamental Diagram of Pedestrian Movement Revisited. Journal of Statistical Mechanics: Theory and Experiment 10:(10).

Verdière, N., Lanza, V., Charrier, R., et al. 2014. Mathematical Modeling of Human Behaviors During Catastrophic Events. Paper presented at ICCSA14, 23-26 June, Le Havre, 8 p.

Verdière, N., Cantin, G., Provitolo, D., et al. 2015. Understanding and Simulation of Human Behaviors in Areas Affected by Disasters: From the Observation to the Conception of a Mathematical Model. Global Journal of Human Social Science: H, Interdisciplinary Volume 15, Issue 10:10 p.

Zhang, X. L., Weng, W. G., Yuan, H. Y., Chen, J. G. 2013. Empirical study of a unidirectional dense crowd during a real mass event. Physica A 392:2781-2791.

Zhou, J.-B., Chen, H., Yang, J., and Yan, J. 2014. Pedestrian Evacuation Time Model for Urban Metro Hubs Based on Multiple Video Sequences Data. Mathematical Problems in Engineering Vol. 2014 Article ID 843096:11 p. 